\title{
Long-term carriage, and transmission of methicillin-resistant Staphylococcus aureus after discharge from hospital
}

\author{
H. M. E. Frénay*†, C. M. J. E. Vandenbroucke-Grauls*, \\ M. J. C. H. Molkenboer $\dagger$ and J. Verhoef* \\ *Utrecht University Hospital, Department of Clinical Microbiology and \\ Laboratory for Infectious Diseases, Utrecht, the Netherlands, and $\dagger$ National \\ Institute of Public Health and Environmental Protection, Bilthoven, the \\ Netherlands
}

Accepted for publication 4 September 1992

\begin{abstract}
Summary: The purpose of this study was to determine whether patients who become carriers of methicillin-resistant Staphylococcus aureus (MRSA) during their stay in hospital, remain colonized after discharge. Thirty-six patients colonized with MRSA during one of three outbreaks at Utrecht University Ilospital (1986-89) were screened 2 or 3 years after they had become carriers. Patients were also interviewed to determine factors contributing to persistent carriage, such as antibiotics, re-admissions to the hospital, presence of skin lesions and chronic diseases. At the same time transmission of MRSA to family members was determined. The epidemic MRSA strain was still found in three patients ( $8 \%$ ). One was a cystic fibrosis patient who had had frequent re-admissions to the hospital and had received several courses of antibiotic treatment. Both of the other patients had skin lesions: a fistula and a colostomy respectively. None of the 44 family members of the patients was colonized or infected with MRSA. We conclude that longterm MRSA carriage occurs with low frequency and is comparable to persistent carriage of methicillin-sensitive Staphylococcus aureus (MSSA). Transmission of MRSA to healthy individuals in an antibiotic-free environment is a rare event.
\end{abstract}

Keywords: Methicillin-resistant Staphylococcus aureus (MRSA); persistent MRSA carrier; transmission to family members.

\section{Introduction}

The incidence of infections due to methicillin-resistant Staphylococcus aureus (MRSA) strains in American and European hospitals has increased over the past 20 years. ${ }^{1,2}$ Also, resistance of MRSA to newer antibiotics, such as quinolones, has been reported with increasing frequency. ${ }^{3}$

Control strategies are advised in order to prevent further spread of

Correspondence to: Hendrina M. E. Frénay, National Institute of Public IIealu and Envirommental Protection, Laboratory of Bacteriology and Antimicrobial Agents, P.O. Box 1, 3720 BA Bilthoven, the Netherlands. 
MRSA in hospitals and nursing homes. ${ }^{4,5}$ While much is known about the behaviour of MRSA in these institutions during outbreaks, follow-up data on the carriage of MRSA in patients who have been discharged from the hospital are not available. Also, little is known about the spread of MRSA strains in the community, particularly to relatives of MRSA-carrying patients. At Utrecht University Hospital three outbreaks of infection due to the same MRSA strain took place between 1986 and 1989, during which 62 patients became colonized or infected. ${ }^{6}$ Every time one of these patients is re-admitted, standard isolation procedures are followed to prevent re-introduction of MRSA into the hospital.

The aim of this study was to determine whether patients remain carriers of MRSA following their discharge. Long-term carriage was defined as carriage for more than 1 year. Therefore, in the period between December 1990 and March 1991 we determined the carriage of MRSA among the patients who had been colonized or infected with MRSA during any of the three MRSA outbreaks. Cultures for bacteriological investigation were taken from these patients at home. To determine possible spread within families, family members were screened for MRSA as well during the same visit.

\section{Patients and methods}

\section{Patients}

A protocol with procedures was approved by the Medical Ethics Committee of Utrecht University Hospital. All patients who had become infected and/or colonized with MRSA during one of the three MRSA outbreaks in 1986, 1987 and 1988/89, were traced through municipal offices. Their medical records were reviewed and the following variables were abstracted: reason for admission to the hospital, treatment of infections with MRSA and treatment of MRSA carriage. During outbreaks patients with MRSA infections had been treated with intravenous vancomycin. The treatment of carriage had consisted of nasal application of neomycin-bacitracin cream. If this treatment had failed a second course with this nasal cream (first outbreak) or with mupirocin (second and third outbreaks) had been prescribed. In addition, a disinfectant soap containing either hexachlorophane, or chlorhexidine, had been used for 7 days. Perineal carriers had been treated with $125 \mathrm{mg}$ vancomycin po 6-hourly in 1987 and 1988/89.

A letter was sent to the patients in which the aim of the study and the procedures to follow were explained. The family members, i.e. those living in the same house, received a separate letter. One member of the study team (H.M.E.F.) made an appointment with the patients and their relatives for the interviews and for taking samples for culture.

Interviews

Interviews focused on four factors that might have played a role in 
persistent carriage of MRSA: (a) antibiotic courses of at least 5 days duration or intravenously administered antibiotics which were prescribed since discharge from the hospital; (b) any admission to a hospital or nursing home since discharge; (c) the presence of skin lesions or skin diseases, either persisting open wounds and stomata or chronic skin diseases such as eczema or psoriasis; (d) the present health status of the patients as compared to their 'original' health status upon admission to the Utrecht University Hospital: (i) total recovery from this disease or (ii) still suffering from the original disease and/or its complications.

\section{Bacteriological investigations}

Cotton-tipped swabs were used to obtain samples for culturing. Cultures were taken of the anterior nares, throat and perineum. If present, skin lesions were also cultured. Family-members were screened for MRSA by nasal swabs only. Specimens were transported to the laboratory within $6 \mathrm{~h}$ after sampling. Direct plating was performed on $5 \%$ sheep blood agar, mannitol salt agar and Mueller-Hinton agar supplemented with $10 \mu \mathrm{g} \mathrm{ml}^{-1}$ methicillin. The swabs were then placed in a staphylococcal enrichment broth supplemented with methicillin $\left(10 \mu \mathrm{g} \mathrm{ml}^{-1}\right)$ to increase sensitivity. ${ }^{7}$ Giolitti-Cantoni broth was used for this purpose. ${ }^{8}$ In this broth potassium tellurite is used as a colour indicator for the presence of $S$. aureus. Methicillin was added to a concentration of $10 \mu \mathrm{g} \mathrm{ml}^{-1}$. Preliminary experiments indicated that less than 10 MRSA colony-forming units (cfu) $\mathrm{ml}^{-1}$ were detectable in this broth. The agar plates and enrichment broth were incubated for $48 \mathrm{~h}$ at $35^{\circ} \mathrm{C}$. Colonies suspected to be $S$. aureus were tested for catalase and tube coagulase. After 24 or $48 \mathrm{~h}$ the broth was subcultured on blood agar if change of colour had taken place. All S. aureus isolates were phage-typed. The international set of phages was used at 100 -fold RTD as well as at RTD. Additional phage typing was performed with a set of Dutch phages ${ }^{9}$ and a set of experimental phages specific for typing MRSA-strains. Resistance to co-amoxiclav and methicillin was tested by determination of minimal inhibitory concentration (MIC) values by agar dilution according to the standards of the National Committee of Clinical Laboratory Standards. Other antibiotics were tested according to the recommendations of the Dutch Working Party on susceptibility testing. ${ }^{10}$

\section{Statistical analysis}

Fisher's exact test was used for statistical analysis.

\section{Results}

Thirty-six patients were still alive at the time of this study; they all agreed to participate ('Table I). These patients were surveyed between 18 months and 3 years after their discharge. Forty-four family members took part, only two 
Table I. Patients enrolled in the MRSA-study

\begin{tabular}{lccccc}
\hline Outbreak & $\begin{array}{c}\text { Involved in } \\
\text { outbreak }\end{array}$ & $\begin{array}{c}\text { Available for } \\
\text { study }\end{array}$ & $\begin{array}{c}\text { Treated for } \\
\text { *infection and } \\
\text { †carriage }\end{array}$ & $\begin{array}{c}\text { Treatcd for } \\
\text { carriage only }\end{array}$ & Not treated \\
& 11 & 5 & 3 & 0 & 2 \\
1986 & 19 & 11 & 4 & 4 & 3 \\
1987 & 32 & 20 & 8 & 6 & 6 \\
Total & 62 & 36 & 15 & 10 & 11 \\
\hline
\end{tabular}

*Infections with MRSA were treated with vancomycin intravenously.

$\dagger$ Carriage of MRSA was treated with nasal creams (neomycin/bacitracin and/or mupirocin), disinfectant soap (hexachlorophane or chlorhexidine) and oral vancomycin (in case of perineal carriage).

family members refused to do so. Six patients lived in Utrecht. The other 30 families lived outside Utrecht up to distances of 150 miles away.

\section{Long-term MRSA-carriers}

Three patients were still carriers of MRSA. In each case, phage typing and antibiogram confirmed the MRSA strain to be identical to the epidemic MRSA strain of the three hospital outbreaks. This strain was not typable with the international set of phages, but could be typed with the experimental set of MRSA phages as $1 / 16 / 23 / 24 / 25 / 26$. The antibiogram showed resistance to methicillin (MIC $>64 \mathrm{mg} \mathrm{l}^{-1}$ ), co-amoxiclav (MIC $>16 \mathrm{mg} \mathrm{l}^{-1}$ ), gentamicin $\left(\mathrm{MIC}>16 \mathrm{mg} \mathrm{l}^{-1}\right.$ ), tetracycline $\left(\mathrm{MIC}>4 \mathrm{mg} \mathrm{l}^{-1}\right.$ ), erythromycin ( $\left.\mathrm{MIC}>4 \mathrm{mg}^{-1}\right)$, clindamycin $\left(\mathrm{MIC}>4 \mathrm{mg} \mathrm{l}^{-1}\right)$, rifampicin $\left(\mathrm{MIC}>1 \mathrm{mg}^{-1}\right)$, intermediate resistance to ciprofloxacin $\left(\mathrm{MIC}=4 \mathrm{mg} \mathrm{l}^{-1}\right.$ ) and amikacin $\left(\mathrm{MIC}=4 \mathrm{mg} \mathrm{l}^{-1}\right)$. It was sensitive to neomycin (MIC $<1 \mathrm{mg} \mathrm{l}^{-1}$ ), fusidic acid (MIC $<1 \mathrm{mg} \mathrm{l}^{-1}$ ), chloramphenicol (MIC $<4 \mathrm{mg} \mathrm{l}^{-1}$ ), co-trimoxazole ( $\mathrm{MIC}<0.12 \mathrm{mg} \mathrm{l}^{-1}$ ), vancomycin $\left(\mathrm{MIC}<2 \mathrm{mg} \mathrm{l}^{-1}\right.$ ) and mupirocin (MIC $<0.12 \mathrm{mg} \mathrm{l}^{-1}$ ). The medical histories of the persistent MRSA carriers and the results of culture of their relatives were as follows.

Patient no. 1: 18-year-old male. This patient was first admitted to Utrecht University Hospital in 1987 bccause of acutc oesophageal bleeding. After surgery he was nursed in the Intensive Care Unit at the time of the second MRSA outbreak. He developed abdominal abscesses with MRSA. Cystic fibrosis was diagnosed as the underlying cause of cirrhosis and oesophageal bleeding. His hospital stay lasted 4 months, during which neomycin-bacitracin and mupirocin nasal creams, hexachlorophane disinfectant soap and oral vancomycin were given for eradication of MRSA. MRSA carriage, however, persisted. Since discharge in October 1987 he was re-admitted several times, during which, treatment with intravenous 
antibiotics was necessary. Each time standard isolation procedures were followed and repeated courses of nasal cream (neomycin-bacitracin and mupirocin) were prescribed, together with chlorhexidine disinfectant soap and oral vancomycin. Despite this treatment, MRSA has been cultured from various body sites such as sputum, sinus secretion and abdominal pus. At the time of the follow-up for this study, MRSA was cultured from his anterior nares, throat and sputum, 27 months after his first discharge. His mother and brother were negative for MRSA while his father carried a methicillin-sensitive $S$, aureus (MSSA) strain.

Patient no. 2: 56-year-old male. This patient was admitted to our hospital in August 1988 with rupture of an abdominal aneurysm. He had a prolonged postoperative course with abdominal complications. $\mathrm{He}$ developed an intravenous-catheter-related bloodstream infection with MRSA during the third MRSA outbreak and was the index patient. MRSA eradication was attempted with nasal creams (neomycin-bacitracin and mupirocin), chlorhexidine soap and oral vancomycin. MRSA was cultured from a decubitus wound up to 2 weeks before he left our hospital. Since his discharge in March 1989 he has not been re-admitted to a hospital. At the time of this follow-up, an abdominal slime fistula was present, which was still productive. Direct plating of all cultures was negative, but the MRSA strain was isolated after $48 \mathrm{~h}$ from the broth culture of his throat specimen. The perineal swab yielded a strain of MSSA which was typable with the international set of phages. MRSA was not isolated from his wife or three children. One daughter carried an MSSA strain, different from the MSSA strain of her father. Hence, patient no. 2 has been a carrier of the epidemic MRSA strain for over 2 years.

Patient no. 3: 68-year-old male. His medical history includcd a colostomy following a rectal amputation (in 1983) and hemiparesis due to a cerebrovascular accident in 1987 . He underwent cardiovascular surgery for aortic valve replacement in January 1989. Postoperatively, he developed a sternal wound infection with MRSA. MRSA was also cultured from his nose and colostomy. Mupirocin nasal cream, chlorhexidine soap and oral vancomycin had been prescribed for 7 days. All cultures had been negative for MRSA during the last 2 days of his stay in hospital.

His nasal swab grew MRSA after $24 \mathrm{~h}$ on agar plates and in broth. A swab taken from the colostomy and a throat swab were both negative for MRSA. At the time of this study he has been a persistent MRSA carrier for 2 years after his discharge from hospital. His wife did not participate in the study.

\section{Families}

In the remaining 33 patients MRSA was not isolated. In this group 37 family members were screened. MRSA was not cultured from any. All family members, including the seven family members of the three persistent 
MRSA carriers, were individuals without a recent or long-term medical history.

In 17 of the 36 families a MSSA strain was isolated from the patient and/or one or more family members. Two such families were families with a persistent MRSA carrier (nos 1 and 2). In three families the patient was colonized with MSSA, and in eight families only one or more members were MSSA carriers. In four families both the patient and family members were found to carry a MSSA strain. Apart from the family of MRSA carrier no. 2 in which two different MSSA strains were found, the same MSSA strain within one family was present as was shown by phage-typing and antibiogram. All MSSA strains were sensitive to the antibiotics tested and to mupirocin.

\section{Interviews}

The results of the interviews are summarized in Table II for the MRSA-positive $(N=3)$ and MRSA-negative $(N=33)$ patients. There were no statistically significant differences between the groups.

\section{Discussion}

Methicillin-resistant $S$. aureus (MRSA) is a well-known and well-studied cause of outbreaks of hospital infection. The rate of MRSA carriage among patients and staff during epidemics and the attack-rate of MRSA have been delineated. ${ }^{411}$ To terminate these outbreaks the necessity for stringent control measures has been stressed.

Carriage of MRSA is prolonged in patients in long-term facilities, where antibiotics are frequently prescribed to those with chronic diseases. ${ }^{12,13}$ The natural history of MRSA carriage in patients that have been infected or colonized with MRSA during a hospital outbreak and return home afterwards, has not been studied to date. The carriage of $S$. aureus and later of penicillin-resistant $S$. aureus has been the subject of considerable research in the 1950-60s. ${ }^{14,15}$ Acquisition of penicillin-resistant strains was

Table II. Risk factors for persistent carriage of $M R S A$

\begin{tabular}{lcc}
\hline Patients & $\begin{array}{c}\text { MRSA-positive } \\
(N=3)\end{array}$ & $\begin{array}{c}\text { MRSA-negative } \\
(N=33)\end{array}$ \\
\hline Antibiotics since discharge (a) & 1 & 18 \\
Re-admissions to hospital (b) & 1 & 9 \\
Skin lesions and diseases (c) & 2 & 12 \\
Persistence of underlying disease (d) & 3 & 10 \\
\hline
\end{tabular}

$P$-values were calculated with Fisher's exact test (a: $P=0.59$; b: $P=1.00$; c: $P=0.55$; d: $P=0.22$ ). 
shown to be enhanced by the administration of antibiotics during the stay of the patient in hospital. ${ }^{16}$ The acquisition of penicillin-sensitive $S$. aureus was more readily followed by a persistent carrier state than acquisition of a penicillin-resistant $S$. aureus in a study among recruits of the Royal Air Force. ${ }^{15}$ Thompson showed that carriage of penicillin-resistant $S$. aureus persisted longer in nurses than carriage of multiply resistant (penicillin, sulphonamide, streptomycin, chloramphenicol, tetracycline resistant) strains. ${ }^{17}$ In 1964, Noble et al. performed a 6-month follow-up study in healthy individuals to determine the rate of $S$. aureus carriage. From the data of this study we recalculated that the rate of persistent MSSA carriage in a normal population is $13 \% .^{18}$

We have performed a study among patients who have been infected or became colonized with the same MRSA strain during one of three outbreaks (1986-89) in our hospital. Three of 36 patients $(8 \%)$ were persistent MRSA carriers. This rate does not differ significantly from the persistent carrier rate for MSSA found by Noble et al. ${ }^{18}$

Interviews were conducted with the patients in an attempt to identify factors which could contribute to the continuing MRSA carriage. Antibiotic courses and re-admissions to hospital have been considered to be such factors. ${ }^{16}$ In a normal population carriers of penicillin-resistant $S$. aureus were found to have more skin diseases than carriers of penicillin-sensitive $S$. aureus strains. ${ }^{18}$ Moreover, chronic debilitating diseases predispose to a high rate of carriage of multiresistant microorganisms. ${ }^{12,19}$ One of the persistent MRSA carriers was a cystic fibrosis patient. Cystic fibrosis patients are known to harbour multiresistant microorganisms, e.g. Pseudomonas, and are often not able to clear these bacteria ${ }^{20}$ During re-admissions to our hospital after his discharge in 1987, this patient has had repeated courses of intravenous antibiotics. The other two persistent MRSA carriers were both involved in the third MRSA outbreak (1988/89). They have not been re-admitted nor have they received antibiotics. Common factors in these two patients were skin lesions and faecal incontinence. In nursing-home patients faecal incontinence is considered as a contributing factor to persistent carriage of multiresistant microorganisms ${ }^{12}$ However, the distribution of risk factors between MRSA carriers and non-carriers was not significantly different.

To reduce the risk of false-negative cultures a selective enrichment broth for all culture specimens was included. For epidemiological surveillance the use of such a broth is advised., ${ }^{7,21,22}$ In one persistent MRSA carrier the MRSA strain was only found in the broth culture of the throat swab. The throat is a known reservoir for recolonization of the anterior nares. ${ }^{23}$

The risk of spread of MRSA to the community is unknown, but is suggested to be low. ${ }^{24}$ In most MRSA outbreaks only $2-5 \%$ of hospital staff are colonized. ${ }^{25}$ This is much lower than the rates reported in outbreaks with MSSA in the 1950s. ${ }^{26}$ Transmission of an epidemic MRSA strain to family members was not revealed in this study. Except for one family 
(persistent MRSA carrier no. 2, who also harboured a MSSA strain), the same MSSA strain was always detected within one family.

We conclude that long-term carriage of MRSA occurs at a low frequency and is comparable to persistent MSSA carriage. Transmission of MRSA to healthy individuals in an antibiotic-free environment is a rare event.

Re-introduction of MRSA into a hospital is likely to occur with the re-admission of a colonized patient or of his contacts in the community. ${ }^{27,28}$ At Utrecht University Hospital we have tagged the medical records of our MRSA-carrying patients and we adhere to standard isolation and screening procedures when they are re-admitted. We also follow these rules for patients transferred to our hospital after a stay in a hospital abroad. So far, we have had no reason to apply these rules to relatives of MRSA patients as well.

We thank W. J. van Leeuwen and J. A. Rost for phage-typing and C. S. Schot for determining the MIC values. A. G. Vilasi is acknowledged for the preparation of the manuscript and J. H. Klokman-Houweling for statistical analysis.

\section{References}

1. Haley RW, Hightower AW, Khabbaz RF et al. The emergence of methicillin-resistant Staphylococcus aureus infections in United Statcs hospitals. Possiblc role of the house staff-patient transfer circuit. Ann Intern Med 1982; 97: 297-308.

2. Maki DG. Institutional methicillin-resistant S. aureus (MRSA)-what can be done. In: Program and Abstracts of the Thirtieth Interscience Conference on Antimicrobial Agents and Chemotherapy. Atlanta, GA: American Society for Microbiology 1990: (Abstr S-64).

3. Maple PAC, Hamilton-Miller JMT, Brumfitt W. World-wide antibiotic resistance in methicillin-resistant Staphylococcus aureus. Lancet 1989; 1: 537-540.

4. Revised guidelines for the control of epidemic methicillin-resistant Staphylococcus aureus. Report of a combined working party of the Hospital Infection Society and British Society for Antimicrobial Chemotherapy (1990). F Hosp Infect 1990; 16: 351-377.

5. Haley RW. Methicillin-resistant Staphylococcus aureus: do we just have to live with it? Ann Intern Med 1991; 114: 162-164.

6. Vandenbroucke-Grauls CM, Frénay HME, van Klingeren B, Savelkoul TF, Verhoef J. Control of epidemic methicillin-resistant Staphylococcus aureus in a Dutch University Hospital. Eur F Clin Microbiol Infect Dis 1991; 10: 6-11.

7. Cookson BD, Webster M, Philips, I. Control of epidemic methicillin-resistant Staphylococcus aureus. Lancet 1987; i: 696

8. Giolotti $G$, Cantoni $C$. A medium for the isolation of staphylococci from foodstuffs. $\mathscr{J}$ Appl Bacteriol 1966; 29: 395-398.

9. Van Leeuwen WJ, Rost JA. An additional set of phages for the typing of Staphylococcus aureus strains of human origin, non-typable with the international basic set of phages. Zentralblatt für Bakteriologie, Parasitenkunde, Infektionskrankheiten und Hygiene 1976; I Abt Suppl 5: 1013-1019.

10. Van Klingeren B, Mouton RP. Standaardisatie van gevoeligheidsbepalingen. Verslag van de Werkgroep Richtlijnen Gevoeligheidsbepalingen ISBN 90-6960-004-8.

11. Duckworth GJ, Lothian JLE, Williams JD. Methicillin-resistant Staphylococcus aureus: report of an outbreak in a London teaching hospital. F Hosp Infect 1988; 11: 1-15.

12. Garibaldi RA, Brodine S, Matsumiya S. Infections among patients in nursing homes. Policies, prevalence and problems. $N$ Engl F Med 1981; 305: 731-735.

13. Muder RR, Brennen C, Wagener MM et al. Methicillin-resistant staphylococcal colonization and infection in a long-term care facility. Ann Intern Med 1991; 114: $107-112$. 
14. Goslings WRO, Büchli K. Nasal carrier rate of antibiotic-resistant staphylococci. $A m$ Med Assoc Arch Int Med 1958; 102: 691-715.

15. Miller DL, McDonald JC, Jevons MP, Williams REO. Staphylococcal disease and nasal carriage in the Royal Air Force. F Hyg, 1962; 60: 451-465.

16. Noble WC, Williams REO, Jevons MP, Shooter RA. Some aspects of nasal carriage of staphylococci. F Clin Pathol 1964; 17: 79-83.

17. Thompson MEM, Gillespie WA. Nasal carriage of Staphylococcus aureus by nurses. $\mathcal{F}$ Pathol Bacteriol 1958; LXXV: 351-355.

18. Noble WC, Valkenburg HA, Wolters CHL. Carriage of Staphylococcus aureus in random samples of a normal population. F Hyg 1967; 65: 567-573.

19. Hsu CCS, Macaluso CP, Special L, Hubble RH. High rate of methicilin resistance of Staphylococcus aureus isolated from hospitalized nursing home patients. Arch Intern Med 1988; 148: 569-570.

20. Høiby N, Koch C. Pseudomonas aeruginosa infection in cystic fibrosis and its management. Thorax 1990; 45: 881-884.

21. Sauter RL, Wells LW. Selective staphylococcal broth. F Clin Microbiol 1990; 28: 23802381 .

22. Cookson BD. Author's reply. F Clin Microbiol 1990; 28: 2381.

23. Casewell MW, Hill RLR. The carrier state: methicillin-resistant Staphylococcus aureus. I Antimicrob Chemother 1986; 18, (Suppl. A): 1-12.

24. Methicillin-resistant Staphylococcus aureus-United States. In: Morbidity and Mortality Weekly Report (MMWR) 1981; 30: 557-559.

25. Cookson B, Peters B, Webster M, Philips I, Rahman M, Noble W. Staff carriage of epidemic methicillin-resistant Staphylococcus aureus. F Clin Microbiol 1989; 27: 1471-1476.

26. Hutchison JGP, Green CA, Grimson TA. Nasal carriage of Staphylococcus aureus in nurses. F Clin Pathol 1957; 10: 92-95.

27. Linnemann CC, Moore P, Stanech J. Reemergence of methicillin-resistant Staphylococcus aureus (MRSA) in a general hospital associated with changing staphylococcal strains (abstract). In: Proceedings of the Third Decennial International Conference on Nosocomial Infections. Atlanta, GA, August 1990.

28. Hicks NR, Moore EP, Williams EW. Carriage and community treatment of methicillin-resistant Staphylococcus aureus: what happens to colonized patients after discharge? F Hosp Infect 1991; 19: 17-24. 